\title{
UBVRI Photometric Analysis of the Solar-Type Eclipsing Binary TYC 3034-299-1
}

\author{
Ronald G. Samec, ${ }^{1,2}$ Adam Jaso,, ${ }^{1,2}$ Jesse White, ${ }^{1,2}$ Danny R. Faulkner, ${ }^{2,3}$ \\ Nicholas Blum, ${ }^{2,3}$ and Walter Van Hamme ${ }^{4}$ \\ ${ }^{1}$ Astronomy Group, Department of Physics and Engineering, Bob Jones University, Greenville, SC 29614, USA \\ ${ }^{2}$ Visiting Astronomer, Lowell Observatory, Flagstaff, AZ 86001, USA \\ ${ }^{3}$ Division Chair of Math, Science, Nursing, and Public health, University of South Carolina, Lancaster, SC 29720, USA \\ ${ }^{4}$ Department of Physics, Florida International University, Miami, FL 33199, USA
}

Correspondence should be addressed to Ronald G. Samec, rsamec@bju.edu

Received 28 November 2011; Accepted 20 December 2011

Academic Editors: P. P. Avelino and S. Bogovalov

Copyright ( $) 2012$ Ronald G. Samec et al. This is an open access article distributed under the Creative Commons Attribution License, which permits unrestricted use, distribution, and reproduction in any medium, provided the original work is properly cited.

TYC 3034-0299-1 $\left(C V_{n}\right)$ is a magnetically active, contact binary, ROTSE variable. $\mathrm{UBVR}_{c} I_{c}$ light curves are presented along with a period study and a simultaneous UBVRI light curve solution. Our light curves show eclipse amplitudes of 0.72 and 0.62 mags $(V)$ in the primary and secondary eclipses. Modeled results include a dark spot region, found at longitude $51^{\circ}$, a $24 \%$ Roche lobe fill-out, and a mass ratio of 0.48. A total eclipse is found to occur in the secondary eclipse making TYC 3034-0299-1 a $W$-type (less massive star is hotter) $W$ UMa variable.

\section{History}

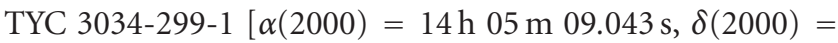
$\left.+38^{\circ} 54^{\prime} 19.26^{\prime \prime}\right]$ was discovered by ROTSE I [1]. It was reported in IBVS no. 5699 [2]. Observations were taken by Diethelm [3]. He gives the following ephemeris:

$$
\mathrm{HJD} T_{\min } \mathrm{I}=2453382.6919+0.395010 d * E .
$$

TYC 3034-299-1 was listed in the catalog of 1022 bright contact binary stars by Geske et al. [4] lists a period of $0.395013 d, V_{\max }=11.462 \mathrm{Vmag}$, and amplitude $=$ $0.585 \mathrm{~V}$ mag. In addition, two times of minimum light were determined by BBSAG observers given in IBVS no. 5781 [3]. Also, two eclipse timings were recently observed by Nelson $[5,6]$.

\section{Finding Chart}

Our comparison star $(C)$ was GSC $30340404[\alpha(2000)=$ $\left.14 \mathrm{~h} 05 \mathrm{~m} 21.4758 \mathrm{~s}, \delta(2000)=+38^{\circ} 48^{\prime} 35.436^{\prime \prime}\right]$. The check star $(K)$ was GSC $30340497[\alpha(2000)=14$ h $05 \mathrm{~m} 13.8544 \mathrm{~s}$, $\left.\delta(2000)=+38^{\circ} 57^{\prime} 24.156^{\prime \prime}\right]$. We include a finding chart of these stars including the variable $(V)$ in Figure 1.

\section{Observations}

This system was observed as a part of our student/professional collaborative studies of interacting binaries from data taken from the national undergraduate research observatory (NURO). The observations were taken by RGS and NB. Reduction and analyses were jointly completed by RGS and AJ. The present 2010 UBVRI light curves were taken with the Lowell $0.81-\mathrm{m}$ reflector in Flagstaff on May 10 and May 11 with CRYOTIGER cooled $\left(-100^{\circ} \mathrm{C}\right)$ 2048 X2048 NASACAM and standard UBVRI Besell filters. The individual observations included 216 in the U-filter, 230 in B, 232 in V, 243 in R, and 239 in I. The standard error of a single observation was $1 \%$ in $\mathrm{U}, \mathrm{V}$, and $\mathrm{R}$ and $\mathrm{I}$ an $1.2 \%$ in $\mathrm{B}$ (Table 1).

\section{Period Determination}

The following timings of minimum light were calculated using parabola fits from our present observations (PO). They were determined in each of UBVRI and averaged. They include (with standard errors) HJD I = $2455326.72754 \pm 0.00024,2455327.713303 \pm 0.00025$; HJD 
Table 1

\begin{tabular}{|c|c|c|c|c|c|c|c|c|c|}
\hline UDM & $\begin{array}{c}\text { UHJD } \\
2455300+\end{array}$ & UDM & $\begin{array}{c}\text { UHJD } \\
2455300+\end{array}$ & UDM & $\begin{array}{c}\text { UHJD } \\
2455300+\end{array}$ & UDM & $\begin{array}{c}\text { UHJD } \\
2455300+\end{array}$ & UDM & $\begin{array}{c}\text { UHJD } \\
2455300+\end{array}$ \\
\hline 0.347 & 26.6640 & 0.402 & 26.7897 & 0.729 & 26.9510 & 0.996 & 27.7258 & 0.312 & 27.8302 \\
\hline 0.370 & 26.6662 & 0.391 & 26.7920 & 0.626 & 26.9555 & 0.959 & 27.7281 & 0.319 & 27.8325 \\
\hline 0.380 & 26.6685 & 0.381 & 26.7960 & 0.459 & 26.9705 & 0.907 & 27.7304 & 0.325 & 27.8353 \\
\hline 0.439 & 26.6796 & 0.381 & 26.7983 & 0.427 & 26.9764 & 0.858 & 27.7329 & 0.339 & 27.8376 \\
\hline 0.459 & 26.6819 & 0.377 & 26.8006 & 0.398 & 26.9810 & 0.801 & 27.7353 & 0.337 & 27.8399 \\
\hline 0.484 & 26.6842 & 0.377 & 26.8043 & 0.392 & 27.6281 & 0.753 & 27.7375 & 0.349 & 27.8423 \\
\hline 0.526 & 26.6873 & 0.361 & 26.8066 & 0.385 & 27.6327 & 0.707 & 27.7401 & 0.358 & 27.8446 \\
\hline 0.547 & 26.6896 & 0.364 & 26.8089 & 0.391 & 27.6363 & 0.668 & 27.7424 & 0.379 & 27.8469 \\
\hline 0.573 & 26.6919 & 0.363 & 26.8123 & 0.397 & 27.6386 & 0.639 & 27.7447 & 0.378 & 27.8507 \\
\hline 0.607 & 26.6945 & 0.354 & 26.8146 & 0.413 & 27.6408 & 0.590 & 27.7474 & 0.388 & 27.8530 \\
\hline 0.641 & 26.6968 & 0.356 & 26.8169 & 0.428 & 27.6439 & 0.560 & 27.7497 & 0.401 & 27.8553 \\
\hline 0.669 & 26.6991 & 0.342 & 26.8200 & 0.433 & 27.6462 & 0.543 & 27.7520 & 0.422 & 27.8587 \\
\hline 0.755 & 26.7031 & 0.352 & 26.8246 & 0.445 & 27.6485 & 0.515 & 27.7546 & 0.431 & 27.8610 \\
\hline 0.803 & 26.7054 & 0.345 & 26.8276 & 0.457 & 27.6512 & 0.491 & 27.7569 & 0.441 & 27.8633 \\
\hline 0.834 & 26.7077 & 0.366 & 26.8299 & 0.473 & 27.6534 & 0.472 & 27.7592 & 0.466 & 27.8665 \\
\hline 0.913 & 26.7112 & 0.342 & 26.8322 & 0.478 & 27.6558 & 0.458 & 27.7615 & 0.478 & 27.8688 \\
\hline 0.923 & 26.7135 & 0.348 & 26.8354 & 0.499 & 27.6600 & 0.410 & 27.7638 & 0.508 & 27.8711 \\
\hline 0.960 & 26.7158 & 0.369 & 26.8377 & 0.506 & 27.6623 & 0.417 & 27.7661 & 0.537 & 27.8743 \\
\hline 0.993 & 26.7190 & 0.375 & 26.8400 & 0.51 & 27.6646 & 0.404 & 27.7685 & 0.554 & 27.8766 \\
\hline 0.997 & 26.7213 & 0.377 & 26.8435 & 0.536 & 27.6689 & 0.400 & 27.7708 & 0.595 & 27.8789 \\
\hline 1.004 & 26.7236 & 0.406 & 26.8458 & 0.572 & 27.6712 & 0.392 & 27.7731 & 0.653 & 27.8820 \\
\hline 0.981 & 26.7268 & 0.416 & 26.8481 & 0.579 & 27.6735 & 0.380 & 27.7757 & 0.677 & 27.8843 \\
\hline 1.007 & 26.7291 & 0.412 & 26.8514 & 0.609 & 27.6763 & 0.365 & 27.7780 & 0.728 & 27.8866 \\
\hline 1.014 & 26.7314 & 0.412 & 26.8537 & 0.641 & 27.6786 & 0.343 & 27.7803 & 0.775 & 27.8890 \\
\hline 0.980 & 26.7351 & 0.423 & 26.8560 & 0.674 & 27.6809 & 0.333 & 27.7851 & 0.802 & 27.8913 \\
\hline 0.994 & 26.7374 & 0.461 & 26.8599 & 0.694 & 27.6840 & 0.334 & 27.7874 & 0.848 & 27.8936 \\
\hline 0.966 & 26.7397 & 0.472 & 26.8623 & 0.741 & 27.6863 & 0.315 & 27.7898 & 0.905 & 27.8970 \\
\hline 0.900 & 26.7439 & 0.456 & 26.8646 & 0.788 & 27.6886 & 0.323 & 27.7921 & 0.946 & 27.8993 \\
\hline 0.859 & 26.7461 & 0.510 & 26.8677 & 0.815 & 27.6909 & 0.312 & 27.7944 & 0.975 & 27.9016 \\
\hline 0.818 & 26.7484 & 0.557 & 26.8782 & 0.869 & 27.6933 & 0.309 & 27.7972 & 1.005 & 27.9047 \\
\hline 0.720 & 26.7540 & 0.582 & 26.8805 & 0.898 & 27.6956 & 0.306 & 27.7995 & 0.992 & 27.9070 \\
\hline 0.696 & 26.7562 & 0.600 & 26.8828 & 0.95 & 27.6979 & 0.292 & 27.8018 & 0.999 & 27.9093 \\
\hline 0.654 & 26.7585 & 0.637 & 26.8864 & 0.997 & 27.7002 & 0.287 & 27.8044 & 1.004 & 27.9117 \\
\hline 0.571 & 26.7629 & 0.674 & 26.8887 & 1.051 & 27.7025 & 0.282 & 27.8067 & 0.992 & 27.9140 \\
\hline 0.534 & 26.7652 & 0.686 & 26.8910 & 1.099 & 27.7049 & 0.283 & 27.8090 & 1.007 & 27.9163 \\
\hline 0.520 & 26.7675 & 0.740 & 26.8948 & 1.126 & 27.7072 & 0.285 & 27.8117 & 0.997 & 27.9188 \\
\hline 0.495 & 26.7713 & 0.791 & 26.8971 & 1.138 & 27.7095 & 0.291 & 27.8140 & 1.007 & 27.9211 \\
\hline 0.487 & 26.7736 & 0.815 & 26.8994 & 1.143 & 27.7119 & 0.288 & 27.8163 & 0.978 & 27.9234 \\
\hline 0.453 & 26.7759 & 0.908 & 26.9031 & 1.14 & 27.7142 & 0.295 & 27.8186 & 0.952 & 27.9262 \\
\hline 0.443 & 26.7792 & 0.968 & 26.9054 & 1.13 & 27.7165 & 0.291 & 27.8210 & 0.899 & 27.9285 \\
\hline 0.426 & 26.7814 & 1.026 & 26.9100 & 1.103 & 27.7189 & 0.303 & 27.8233 & 0.868 & 27.9308 \\
\hline 0.422 & 26.7837 & 1.073 & 26.9123 & 1.08 & 27.7212 & 0.297 & 27.8256 & 0.806 & 27.9336 \\
\hline \multirow[t]{2}{*}{0.410} & 26.7873 & 1.122 & 26.9146 & 1.061 & 27.7235 & 0.314 & 27.8279 & 0.78 & 27.9359 \\
\hline & & & & & & & & 0.733 & 27.9383 \\
\hline \multirow[t]{2}{*}{$\mathrm{BDM}$} & BHJD & BDM & BHJD & BDM & BHJD & BDM & BHJD & BDM & BHJD \\
\hline & $2455300+$ & & $2455300+$ & & $2455300+$ & & $2455300+$ & & $2455300+$ \\
\hline 0.257 & 26.6557 & 0.332 & 26.7844 & 1.027 & 26.9185 & 0.761 & 27.7311 & 0.231 & 27.8360 \\
\hline 0.262 & 26.6580 & 0.330 & 26.7880 & 0.937 & 26.9362 & 0.735 & 27.7336 & 0.245 & 27.8383 \\
\hline 0.281 & 26.6603 & 0.320 & 26.7903 & 0.894 & 26.9385 & 0.689 & 27.7359 & 0.248 & 27.8406 \\
\hline
\end{tabular}


Table 1: Continued.

\begin{tabular}{|c|c|c|c|c|c|c|c|c|c|}
\hline UDM & $\begin{array}{c}\text { UHJD } \\
2455300+\end{array}$ & UDM & $\begin{array}{c}\text { UHJD } \\
2455300+\end{array}$ & UDM & $\begin{array}{c}\text { UHJD } \\
2455300+\end{array}$ & UDM & $\begin{array}{c}\text { UHJD } \\
2455300+\end{array}$ & UDM & $\begin{array}{c}\text { UHJD } \\
2455300+\end{array}$ \\
\hline 0.288 & 26.6646 & 0.320 & 26.7926 & 0.623 & 26.9539 & 0.660 & 27.7382 & 0.26 & 27.8430 \\
\hline 0.318 & 26.6669 & 0.311 & 26.7966 & 0.333 & 26.9770 & 0.625 & 27.7408 & 0.262 & 27.8453 \\
\hline 0.309 & 26.6691 & 0.297 & 26.7989 & 0.294 & 27.6334 & 0.593 & 27.7431 & 0.28 & 27.8476 \\
\hline 0.386 & 26.6802 & 0.297 & 26.8012 & 0.296 & 27.6370 & 0.555 & 27.7454 & 0.295 & 27.8514 \\
\hline 0.391 & 26.6825 & 0.285 & 26.8049 & 0.305 & 27.6393 & 0.525 & 27.7481 & 0.321 & 27.8537 \\
\hline 0.428 & 26.6848 & 0.278 & 26.8072 & 0.33 & 27.6416 & 0.483 & 27.7504 & 0.338 & 27.8560 \\
\hline 0.446 & 26.6879 & 0.262 & 26.8095 & 0.336 & 27.6446 & 0.470 & 27.7527 & 0.331 & 27.8594 \\
\hline 0.474 & 26.6902 & 0.265 & 26.8130 & 0.34 & 27.6469 & 0.425 & 27.7553 & 0.355 & 27.8617 \\
\hline 0.507 & 26.6925 & 0.269 & 26.8153 & 0.345 & 27.6492 & 0.383 & 27.7576 & 0.385 & 27.8640 \\
\hline 0.547 & 26.6952 & 0.269 & 26.8176 & 0.369 & 27.6519 & 0.374 & 27.7599 & 0.4 & 27.8672 \\
\hline 0.567 & 26.6975 & 0.269 & 26.8206 & 0.367 & 27.6542 & 0.365 & 27.7622 & 0.436 & 27.8695 \\
\hline 0.593 & 26.6997 & 0.274 & 26.8230 & 0.378 & 27.6564 & 0.333 & 27.7645 & 0.451 & 27.8718 \\
\hline 0.669 & 26.7037 & 0.278 & 26.8253 & 0.405 & 27.6607 & 0.323 & 27.7668 & 0.485 & 27.8750 \\
\hline 0.713 & 26.7060 & 0.269 & 26.8282 & 0.425 & 27.6630 & 0.321 & 27.7692 & 0.512 & 27.8773 \\
\hline 0.743 & 26.7083 & 0.253 & 26.8305 & 0.429 & 27.6653 & 0.305 & 27.7715 & 0.543 & 27.8796 \\
\hline 0.808 & 26.7119 & 0.255 & 26.8328 & 0.456 & 27.6696 & 0.278 & 27.7738 & 0.6 & 27.8827 \\
\hline 0.832 & 26.7142 & 0.285 & 26.8360 & 0.491 & 27.6719 & 0.277 & 27.7764 & 0.634 & 27.8850 \\
\hline 0.880 & 26.7165 & 0.276 & 26.8383 & 0.504 & 27.6742 & 0.267 & 27.7787 & 0.677 & 27.8873 \\
\hline 0.887 & 26.7196 & 0.284 & 26.8441 & 0.539 & 27.6770 & 0.264 & 27.7810 & 0.711 & 27.8897 \\
\hline 0.886 & 26.7219 & 0.320 & 26.8464 & 0.556 & 27.6793 & 0.250 & 27.7835 & 0.738 & 27.8920 \\
\hline 0.896 & 26.7242 & 0.302 & 26.8487 & 0.597 & 27.6816 & 0.226 & 27.7858 & 0.779 & 27.8943 \\
\hline 0.891 & 26.7275 & 0.320 & 26.8520 & 0.635 & 27.6847 & 0.226 & 27.7881 & 0.842 & 27.8977 \\
\hline 0.900 & 26.7298 & 0.320 & 26.8543 & 0.646 & 27.6870 & 0.212 & 27.7905 & 0.894 & 27.9000 \\
\hline 0.893 & 26.7320 & 0.331 & 26.8566 & 0.699 & 27.6893 & 0.215 & 27.7928 & 0.906 & 27.9023 \\
\hline 0.892 & 26.7358 & 0.356 & 26.8606 & 0.735 & 27.6916 & 0.212 & 27.7951 & 0.94 & 27.9054 \\
\hline 0.871 & 26.7381 & 0.384 & 26.8652 & 0.774 & 27.6939 & 0.195 & 27.7979 & 0.922 & 27.9077 \\
\hline 0.844 & 26.7404 & 0.382 & 26.8683 & 0.817 & 27.6962 & 0.202 & 27.8002 & 0.928 & 27.9124 \\
\hline 0.777 & 26.7445 & 0.462 & 26.8788 & 0.851 & 27.6986 & 0.194 & 27.8025 & 0.91 & 27.9147 \\
\hline 0.732 & 26.7468 & 0.476 & 26.8811 & 0.894 & 27.7009 & 0.181 & 27.8051 & 0.922 & 27.9170 \\
\hline 0.696 & 26.7491 & 0.499 & 26.8835 & 0.941 & 27.7032 & 0.180 & 27.8074 & 0.916 & 27.9195 \\
\hline 0.617 & 26.7546 & 0.526 & 26.8870 & 0.989 & 27.7056 & 0.182 & 27.8097 & 0.898 & 27.9218 \\
\hline 0.580 & 26.7569 & 0.542 & 26.8893 & 0.988 & 27.7079 & 0.181 & 27.8124 & 0.887 & 27.9241 \\
\hline 0.559 & 26.7592 & 0.607 & 26.8916 & 0.993 & 27.7102 & 0.202 & 27.8147 & 0.838 & 27.9269 \\
\hline 0.497 & 26.7635 & 0.610 & 26.8954 & 0.974 & 27.7126 & 0.197 & 27.8170 & 0.805 & 27.9292 \\
\hline 0.461 & 26.7658 & 0.684 & 26.8977 & 1.003 & 27.7149 & 0.196 & 27.8194 & 0.74 & 27.9315 \\
\hline 0.444 & 26.7681 & 0.716 & 26.9000 & 0.98 & 27.7172 & 0.205 & 27.8217 & 0.716 & 27.9343 \\
\hline 0.412 & 26.7719 & 0.755 & 26.9037 & 0.996 & 27.7196 & 0.214 & 27.8240 & 0.674 & 27.9366 \\
\hline 0.414 & 26.7742 & 0.875 & 26.9060 & 0.954 & 27.7219 & 0.214 & 27.8263 & 0.641 & 27.9390 \\
\hline 0.378 & 26.7765 & 0.872 & 26.9083 & 0.918 & 27.7242 & 0.199 & 27.8286 & & \\
\hline 0.363 & 26.7798 & 0.887 & 26.9106 & 0.857 & 27.7265 & 0.216 & 27.8309 & & \\
\hline 0.355 & 26.7821 & 0.956 & 26.9129 & 0.826 & 27.7288 & 0.230 & 27.8332 & & \\
\hline VDM & VHJD & VDM & VHJD & VDM & VHJD & VDM & VHJD & VDM & VHJD \\
\hline $2455300+$ & & & $2455300+$ & & $2455300+$ & & $2455300+$ & & $2455300+$ \\
\hline 0.192 & 26.6461 & 0.283 & 26.7907 & 0.781 & 26.9366 & 0.897 & 27.7152 & 0.18 & 27.8220 \\
\hline 0.227 & 26.6561 & 0.271 & 26.7930 & 0.803 & 26.9389 & 0.896 & 27.7175 & 0.185 & 27.8243 \\
\hline 0.234 & 26.6584 & 0.268 & 26.7970 & 0.679 & 26.9449 & 0.888 & 27.7199 & 0.182 & 27.8266 \\
\hline 0.249 & 26.6606 & 0.263 & 26.7993 & 0.53 & 26.9520 & 0.851 & 27.7222 & 0.194 & 27.8289 \\
\hline 0.256 & 26.6650 & 0.249 & 26.8016 & 0.515 & 26.9543 & 0.823 & 27.7245 & 0.2 & 27.8312 \\
\hline 0.266 & 26.6672 & 0.253 & 26.8053 & 0.402 & 26.9610 & 0.785 & 27.7268 & 0.213 & 27.8335 \\
\hline
\end{tabular}


Table 1: Continued.

\begin{tabular}{|c|c|c|c|c|c|c|c|c|c|}
\hline UDM & $\begin{array}{c}\text { UHJD } \\
2455300+\end{array}$ & UDM & $\begin{array}{c}\text { UHJD } \\
2455300+\end{array}$ & UDM & $\begin{array}{c}\text { UHJD } \\
2455300+\end{array}$ & UDM & $\begin{array}{c}\text { UHJD } \\
2455300+\end{array}$ & UDM & $\begin{array}{c}\text { UHJD } \\
2455300+\end{array}$ \\
\hline 0.290 & 26.6695 & 0.252 & 26.8076 & 0.397 & 26.9633 & 0.735 & 27.7291 & 0.199 & 27.8363 \\
\hline 0.347 & 26.6806 & 0.221 & 26.8099 & 0.328 & 26.9715 & 0.705 & 27.7314 & 0.223 & 27.8386 \\
\hline 0.357 & 26.6829 & 0.239 & 26.8133 & 0.276 & 26.9820 & 0.655 & 27.7339 & 0.233 & 27.8409 \\
\hline 0.372 & 26.6852 & 0.218 & 26.8156 & 0.252 & 26.9867 & 0.619 & 27.7362 & 0.24 & 27.8433 \\
\hline 0.402 & 26.6883 & 0.211 & 26.8179 & 0.245 & 26.9890 & 0.585 & 27.7385 & 0.24 & 27.8456 \\
\hline 0.434 & 26.6906 & 0.212 & 26.8210 & 0.231 & 27.6250 & 0.506 & 27.7414 & 0.247 & 27.8479 \\
\hline 0.453 & 26.6929 & 0.226 & 26.8233 & 0.227 & 27.6291 & 0.516 & 27.7434 & 0.273 & 27.8517 \\
\hline 0.490 & 26.6955 & 0.217 & 26.8256 & 0.247 & 27.6314 & 0.492 & 27.7457 & 0.293 & 27.8540 \\
\hline 0.517 & 26.6978 & 0.212 & 26.8286 & 0.248 & 27.6337 & 0.452 & 27.7484 & 0.32 & 27.8563 \\
\hline 0.554 & 26.7001 & 0.220 & 26.8309 & 0.258 & 27.6373 & 0.427 & 27.7507 & 0.327 & 27.8597 \\
\hline 0.612 & 26.7041 & 0.229 & 26.8332 & 0.256 & 27.6396 & 0.399 & 27.7530 & 0.344 & 27.8620 \\
\hline 0.662 & 26.7064 & 0.234 & 26.8364 & 0.276 & 27.6418 & 0.377 & 27.7556 & 0.354 & 27.8643 \\
\hline 0.700 & 26.7087 & 0.234 & 26.8387 & 0.283 & 27.6449 & 0.349 & 27.7579 & 0.384 & 27.8675 \\
\hline 0.754 & 26.7122 & 0.254 & 26.8445 & 0.289 & 27.6472 & 0.336 & 27.7602 & 0.411 & 27.8698 \\
\hline 0.794 & 26.7146 & 0.244 & 26.8468 & 0.295 & 27.6495 & 0.317 & 27.7625 & 0.438 & 27.8721 \\
\hline 0.816 & 26.7169 & 0.259 & 26.8491 & 0.316 & 27.6522 & 0.315 & 27.7648 & 0.465 & 27.8753 \\
\hline 0.826 & 26.7200 & 0.276 & 26.8524 & 0.328 & 27.6545 & 0.284 & 27.7671 & 0.49 & 27.8776 \\
\hline 0.830 & 26.7223 & 0.292 & 26.8547 & 0.333 & 27.6567 & 0.275 & 27.7695 & 0.531 & 27.8799 \\
\hline 0.829 & 26.7246 & 0.284 & 26.8570 & 0.364 & 27.6610 & 0.265 & 27.7718 & 0.559 & 27.8830 \\
\hline 0.834 & 26.7278 & 0.314 & 26.8609 & 0.36 & 27.6633 & 0.254 & 27.7741 & 0.622 & 27.8853 \\
\hline 0.817 & 26.7301 & 0.317 & 26.8633 & 0.367 & 27.6656 & 0.233 & 27.7767 & 0.643 & 27.8876 \\
\hline 0.835 & 26.7324 & 0.303 & 26.8656 & 0.407 & 27.6699 & 0.235 & 27.7790 & 0.685 & 27.8900 \\
\hline 0.826 & 26.7361 & 0.319 & 26.8687 & 0.43 & 27.6722 & 0.228 & 27.7813 & 0.723 & 27.8923 \\
\hline 0.823 & 26.7384 & 0.413 & 26.8792 & 0.409 & 27.6748 & 0.214 & 27.7838 & 0.788 & 27.8946 \\
\hline 0.775 & 26.7407 & 0.395 & 26.8815 & 0.475 & 27.6773 & 0.208 & 27.7861 & 0.794 & 27.8980 \\
\hline 0.711 & 26.7449 & 0.453 & 26.8838 & 0.488 & 27.6796 & 0.191 & 27.7884 & 0.823 & 27.9003 \\
\hline 0.674 & 26.7472 & 0.452 & 26.8874 & 0.509 & 27.6819 & 0.198 & 27.7908 & 0.826 & 27.9027 \\
\hline 0.646 & 26.7495 & 0.487 & 26.8897 & 0.565 & 27.6850 & 0.196 & 27.7931 & 0.853 & 27.9057 \\
\hline 0.561 & 26.7550 & 0.535 & 26.8920 & 0.597 & 27.6873 & 0.187 & 27.7954 & 0.842 & 27.9080 \\
\hline 0.528 & 26.7572 & 0.546 & 26.8958 & 0.616 & 27.6896 & 0.178 & 27.7982 & 0.844 & 27.9103 \\
\hline 0.500 & 26.7596 & 0.667 & 26.9004 & 0.65 & 27.6919 & 0.179 & 27.7982 & 0.846 & 27.9127 \\
\hline 0.437 & 26.7639 & 0.705 & 26.9041 & 0.696 & 27.6942 & 0.179 & 27.8005 & 0.868 & 27.9150 \\
\hline 0.414 & 26.7662 & 0.746 & 26.9064 & 0.739 & 27.6965 & 0.180 & 27.8028 & 0.847 & 27.9173 \\
\hline 0.403 & 26.7685 & 0.772 & 26.9086 & 0.78 & 27.6989 & 0.171 & 27.8054 & 0.844 & 27.9198 \\
\hline 0.357 & 26.7723 & 0.829 & 26.9110 & 0.823 & 27.7012 & 0.169 & 27.8077 & 0.802 & 27.9221 \\
\hline 0.335 & 26.7746 & 0.871 & 26.9133 & 0.855 & 27.7035 & 0.164 & 27.8100 & 0.721 & 27.9244 \\
\hline 0.342 & 26.7769 & 0.871 & 26.9189 & 0.888 & 27.7059 & 0.176 & 27.8127 & 0.684 & 27.9295 \\
\hline 0.313 & 26.7802 & 0.908 & 26.9212 & 0.882 & 27.7082 & 0.170 & 27.8150 & 0.643 & 27.9318 \\
\hline 0.317 & 26.7824 & 0.875 & 26.9235 & 0.898 & 27.7105 & 0.186 & 27.8173 & 0.605 & 27.9346 \\
\hline 0.289 & 26.7847 & 0.919 & 26.9260 & 0.914 & 27.7129 & 0.192 & 27.8197 & 0.58 & 27.9369 \\
\hline 0.285 & 26.7883 & 0.870 & 26.9306 & & & & & & \\
\hline \multirow[t]{2}{*}{ RDM } & RHJD & RDM & RHJD & $\mathrm{RDM}$ & RHJD & RDM & RHJD & $\mathrm{RDM}$ & RHJD \\
\hline & $2455300+$ & & $2455300+$ & & $2455300+$ & & $2455300+$ & & $2455300+$ \\
\hline 0.195 & 26.6564 & 0.229 & 26.7973 & 0.454 & 26.9523 & 0.835 & 27.7108 & 0.155 & 27.8269 \\
\hline 0.209 & 26.6587 & 0.219 & 26.7996 & 0.44 & 26.9568 & 0.839 & 27.7132 & 0.167 & 27.8292 \\
\hline 0.216 & 26.6609 & 0.230 & 26.8019 & 0.365 & 26.9636 & 0.832 & 27.7155 & 0.177 & 27.8315 \\
\hline 0.239 & 26.6652 & 0.197 & 26.8056 & 0.278 & 26.9717 & 0.828 & 27.7178 & 0.176 & 27.8338 \\
\hline 0.240 & 26.6675 & 0.217 & 26.8079 & 0.267 & 26.9740 & 0.811 & 27.7202 & 0.183 & 27.8366 \\
\hline 0.241 & 26.6698 & 0.209 & 26.8102 & 0.235 & 26.9823 & 0.807 & 27.7225 & 0.195 & 27.8389 \\
\hline
\end{tabular}


Table 1: Continued.

\begin{tabular}{|c|c|c|c|c|c|c|c|c|c|}
\hline UDM & $\begin{array}{c}\text { UHJD } \\
2455300+\end{array}$ & UDM & $\begin{array}{c}\text { UHJD } \\
2455300+\end{array}$ & UDM & $\begin{array}{c}\text { UHJD } \\
2455300+\end{array}$ & UDM & $\begin{array}{c}\text { UHJD } \\
2455300+\end{array}$ & UDM & $\begin{array}{c}\text { UHJD } \\
2455300+\end{array}$ \\
\hline 0.317 & 26.6809 & 0.200 & 26.8136 & 0.232 & 26.9870 & 0.774 & 27.7248 & 0.194 & 27.8412 \\
\hline 0.333 & 26.6832 & 0.186 & 26.8159 & 0.212 & 26.9893 & 0.736 & 27.7271 & 0.21 & 27.8436 \\
\hline 0.347 & 26.6855 & 0.185 & 26.8182 & 0.252 & 26.9740 & 0.696 & 27.7294 & 0.208 & 27.8459 \\
\hline 0.380 & 26.6886 & 0.188 & 26.8213 & 0.241 & 26.9823 & 0.652 & 27.7317 & 0.224 & 27.8482 \\
\hline 0.392 & 26.6909 & 0.188 & 26.8236 & 0.233 & 26.9870 & 0.606 & 27.7342 & 0.258 & 27.8520 \\
\hline 0.417 & 26.6932 & 0.183 & 26.8259 & 0.22 & 26.9893 & 0.575 & 27.7365 & 0.254 & 27.8543 \\
\hline 0.461 & 26.6958 & 0.181 & 26.8289 & 0.838 & 26.9270 & 0.527 & 27.7388 & 0.255 & 27.8566 \\
\hline 0.492 & 26.6981 & 0.194 & 26.8312 & 0.805 & 26.9293 & 0.465 & 27.7437 & 0.271 & 27.8600 \\
\hline 0.524 & 26.7004 & 0.180 & 26.8335 & 0.716 & 26.9344 & 0.435 & 27.7460 & 0.294 & 27.8623 \\
\hline 0.583 & 26.7044 & 0.204 & 26.8367 & 0.674 & 26.9367 & 0.402 & 27.7487 & 0.295 & 27.8646 \\
\hline 0.613 & 26.7066 & 0.189 & 26.8390 & 0.641 & 26.9390 & 0.384 & 27.7510 & 0.328 & 27.8678 \\
\hline 0.656 & 26.7090 & 0.210 & 26.8448 & 0.19 & 27.6294 & 0.357 & 27.7533 & 0.343 & 27.8701 \\
\hline 0.714 & 26.7125 & 0.214 & 26.8471 & 0.19 & 27.6316 & 0.337 & 27.7559 & 0.36 & 27.8724 \\
\hline 0.735 & 26.7148 & 0.236 & 26.8494 & 0.215 & 27.6339 & 0.316 & 27.7582 & 0.403 & 27.8756 \\
\hline 0.773 & 26.7171 & 0.225 & 26.8526 & 0.218 & 27.6376 & 0.303 & 27.7605 & 0.416 & 27.8779 \\
\hline 0.790 & 26.7202 & 0.231 & 26.8550 & 0.215 & 27.6398 & 0.280 & 27.7628 & 0.437 & 27.8802 \\
\hline 0.784 & 26.7225 & 0.247 & 26.8573 & 0.224 & 27.6421 & 0.268 & 27.7651 & 0.499 & 27.8833 \\
\hline 0.778 & 26.7249 & 0.281 & 26.8659 & 0.246 & 27.6452 & 0.253 & 27.7674 & 0.531 & 27.8856 \\
\hline 0.784 & 26.7281 & 0.297 & 26.8690 & 0.248 & 27.6475 & 0.246 & 27.7698 & 0.557 & 27.8879 \\
\hline 0.792 & 26.7304 & 0.349 & 26.8795 & 0.254 & 27.6497 & 0.239 & 27.7721 & 0.59 & 27.8903 \\
\hline 0.770 & 26.7327 & 0.373 & 26.8818 & 0.258 & 27.6525 & 0.228 & 27.7744 & 0.633 & 27.8926 \\
\hline 0.780 & 26.7364 & 0.400 & 26.8841 & 0.271 & 27.6547 & 0.222 & 27.7770 & 0.678 & 27.8949 \\
\hline 0.773 & 26.7387 & 0.402 & 26.8877 & 0.273 & 27.6570 & 0.201 & 27.7793 & 0.718 & 27.8983 \\
\hline 0.725 & 26.7410 & 0.440 & 26.8900 & 0.298 & 27.6613 & 0.195 & 27.7816 & 0.76 & 27.9006 \\
\hline 0.662 & 26.7452 & 0.463 & 26.8923 & 0.309 & 27.6636 & 0.190 & 27.7841 & 0.793 & 27.9029 \\
\hline 0.627 & 26.7474 & 0.518 & 26.8961 & 0.317 & 27.6659 & 0.182 & 27.7864 & 0.783 & 27.9060 \\
\hline 0.592 & 26.7497 & 0.549 & 26.8984 & 0.346 & 27.6702 & 0.169 & 27.7887 & 0.788 & 27.9083 \\
\hline 0.509 & 26.7552 & 0.586 & 26.9007 & 0.37 & 27.6725 & 0.171 & 27.7911 & 0.79 & 27.9106 \\
\hline 0.486 & 26.7575 & 0.629 & 26.9044 & 0.417 & 27.6776 & 0.160 & 27.7934 & 0.796 & 27.9130 \\
\hline 0.461 & 26.7598 & 0.716 & 26.9067 & 0.443 & 27.6799 & 0.160 & 27.7957 & 0.785 & 27.9153 \\
\hline 0.407 & 26.7642 & 0.727 & 26.9089 & 0.457 & 27.6822 & 0.151 & 27.7985 & 0.795 & 27.9176 \\
\hline 0.376 & 26.7665 & 0.752 & 26.9113 & 0.516 & 27.6853 & 0.143 & 27.8008 & 0.768 & 27.9201 \\
\hline 0.348 & 26.7688 & 0.799 & 26.9136 & 0.532 & 27.6876 & 0.150 & 27.8031 & 0.783 & 27.9224 \\
\hline 0.329 & 26.7726 & 0.848 & 26.9191 & 0.571 & 27.6899 & 0.137 & 27.8057 & 0.747 & 27.9247 \\
\hline 0.318 & 26.7749 & 0.822 & 26.9214 & 0.606 & 27.6922 & 0.141 & 27.8080 & 0.712 & 27.9275 \\
\hline 0.311 & 26.7772 & 0.848 & 26.9237 & 0.637 & 27.6945 & 0.144 & 27.8103 & 0.673 & 27.9298 \\
\hline 0.279 & 26.7805 & 0.832 & 26.9262 & 0.682 & 27.6968 & 0.147 & 27.8130 & 0.632 & 27.9321 \\
\hline 0.266 & 26.7827 & 0.838 & 26.9285 & 0.718 & 27.6992 & 0.151 & 27.8153 & 0.597 & 27.9349 \\
\hline 0.255 & 26.7850 & 0.808 & 26.9308 & 0.755 & 27.7015 & 0.152 & 27.8176 & 0.545 & 27.9372 \\
\hline 0.245 & 26.7887 & 0.777 & 26.9346 & 0.785 & 27.7038 & 0.156 & 27.8199 & 0.536 & 27.9395 \\
\hline 0.240 & 26.7909 & 0.750 & 26.9369 & 0.818 & 27.7061 & 0.140 & 27.8222 & & \\
\hline 0.242 & 26.7933 & 0.732 & 26.9392 & 0.83 & 27.7084 & 0.155 & 27.8246 & & \\
\hline \multirow[t]{2}{*}{ IDM } & IHJD & IDM & IHJD & IDM & IHJD & IDM & IHJD & IDM & IHJD \\
\hline & $2455300+$ & & $2455300+$ & & $2455300+$ & & $2455300+$ & & $2455300+$ \\
\hline 0.181 & 26.6566 & 0.222 & 26.7912 & 0.649 & 26.9395 & 0.769 & 27.7205 & 0.141 & 27.8272 \\
\hline 0.182 & 26.6589 & 0.207 & 26.7935 & 0.629 & 26.9432 & 0.748 & 27.7228 & 0.142 & 27.8295 \\
\hline 0.186 & 26.6612 & 0.189 & 26.7975 & 0.446 & 26.9526 & 0.718 & 27.7251 & 0.148 & 27.8318 \\
\hline 0.206 & 26.6655 & 0.187 & 26.7998 & 0.362 & 26.9571 & 0.686 & 27.7274 & 0.15 & 27.8341 \\
\hline 0.221 & 26.6678 & 0.186 & 26.8022 & 0.258 & 26.9697 & 0.644 & 27.7297 & 0.175 & 27.8369 \\
\hline 0.271 & 26.6812 & 0.183 & 26.8059 & 0.241 & 26.9743 & 0.616 & 27.7320 & 0.16 & 27.8392 \\
\hline
\end{tabular}


Table 1: Continued.

\begin{tabular}{|c|c|c|c|c|c|c|c|c|c|}
\hline UDM & $\begin{array}{c}\text { UHJD } \\
2455300+\end{array}$ & UDM & $\begin{array}{c}\text { UHJD } \\
2455300+\end{array}$ & UDM & $\begin{array}{c}\text { UHJD } \\
2455300+\end{array}$ & UDM & $\begin{array}{c}\text { UHJD } \\
2455300+\end{array}$ & UDM & $\begin{array}{c}\text { UHJD } \\
2455300+\end{array}$ \\
\hline 0.308 & 26.6835 & 0.171 & 26.8082 & 0.232 & 26.9826 & 0.565 & 27.7345 & 0.17 & 27.8415 \\
\hline 0.318 & 26.6858 & 0.167 & 26.8105 & 0.74 & 26.9316 & 0.541 & 27.7368 & 0.198 & 27.8439 \\
\hline 0.345 & 26.6889 & 0.160 & 26.8139 & 0.716 & 26.9344 & 0.502 & 27.7391 & 0.19 & 27.8462 \\
\hline 0.353 & 26.6911 & 0.139 & 26.8162 & 0.674 & 26.9367 & 0.461 & 27.7417 & 0.194 & 27.8485 \\
\hline 0.402 & 26.6935 & 0.148 & 26.8185 & 0.641 & 26.9390 & 0.434 & 27.7440 & 0.233 & 27.8522 \\
\hline 0.430 & 26.6961 & 0.147 & 26.8216 & 0.156 & 27.6296 & 0.401 & 27.7463 & 0.235 & 27.8545 \\
\hline 0.462 & 26.6984 & 0.156 & 26.8239 & 0.165 & 27.6319 & 0.378 & 27.7490 & 0.225 & 27.8569 \\
\hline 0.473 & 26.7007 & 0.157 & 26.8262 & 0.167 & 27.6342 & 0.346 & 27.7513 & 0.25 & 27.8603 \\
\hline 0.538 & 26.7047 & 0.153 & 26.8291 & 0.176 & 27.6379 & 0.328 & 27.7536 & 0.262 & 27.8626 \\
\hline 0.589 & 26.7069 & 0.160 & 26.8314 & 0.174 & 27.6401 & 0.302 & 27.7562 & 0.272 & 27.8649 \\
\hline 0.618 & 26.7092 & 0.148 & 26.8337 & 0.181 & 27.6424 & 0.278 & 27.7585 & 0.287 & 27.8681 \\
\hline 0.662 & 26.7128 & 0.189 & 26.8370 & 0.194 & 27.6455 & 0.264 & 27.7608 & 0.312 & 27.8704 \\
\hline 0.699 & 26.7151 & 0.168 & 26.8393 & 0.221 & 27.6477 & 0.237 & 27.7631 & 0.333 & 27.8727 \\
\hline 0.724 & 26.7174 & 0.145 & 26.8416 & 0.221 & 27.6500 & 0.234 & 27.7654 & 0.355 & 27.8759 \\
\hline 0.737 & 26.7205 & 0.197 & 26.8450 & 0.248 & 27.6527 & 0.223 & 27.7677 & 0.382 & 27.8782 \\
\hline 0.731 & 26.7228 & 0.180 & 26.8496 & 0.245 & 27.6550 & 0.213 & 27.7701 & 0.456 & 27.8805 \\
\hline 0.751 & 26.7251 & 0.207 & 26.8529 & 0.245 & 27.6573 & 0.218 & 27.7724 & 0.472 & 27.8835 \\
\hline 0.727 & 26.7284 & 0.209 & 26.8552 & 0.262 & 27.6616 & 0.198 & 27.7747 & 0.481 & 27.8858 \\
\hline 0.725 & 26.7307 & 0.217 & 26.8575 & 0.28 & 27.6639 & 0.188 & 27.7773 & 0.517 & 27.8881 \\
\hline 0.721 & 26.7330 & 0.229 & 26.8615 & 0.291 & 27.6661 & 0.177 & 27.7796 & 0.557 & 27.8906 \\
\hline 0.722 & 26.7367 & 0.223 & 26.8638 & 0.326 & 27.6705 & 0.174 & 27.7819 & 0.6 & 27.8929 \\
\hline 0.706 & 26.7390 & 0.224 & 26.8661 & 0.341 & 27.6728 & 0.162 & 27.7844 & 0.631 & 27.8952 \\
\hline 0.670 & 26.7413 & 0.315 & 26.8798 & 0.348 & 27.6751 & 0.158 & 27.7867 & 0.698 & 27.8986 \\
\hline 0.625 & 26.7454 & 0.340 & 26.8821 & 0.383 & 27.6778 & 0.156 & 27.7890 & 0.711 & 27.9009 \\
\hline 0.588 & 26.7477 & 0.351 & 26.8844 & 0.434 & 27.6825 & 0.161 & 27.7913 & 0.743 & 27.9032 \\
\hline 0.554 & 26.7500 & 0.375 & 26.8880 & 0.468 & 27.6856 & 0.140 & 27.7937 & 0.747 & 27.9063 \\
\hline 0.483 & 26.7555 & 0.412 & 26.8903 & 0.502 & 27.6879 & 0.130 & 27.7960 & 0.715 & 27.9086 \\
\hline 0.441 & 26.7578 & 0.446 & 26.8926 & 0.53 & 27.6902 & 0.129 & 27.7988 & 0.756 & 27.9109 \\
\hline 0.402 & 26.7601 & 0.511 & 26.8963 & 0.562 & 27.6925 & 0.125 & 27.8011 & 0.76 & 27.9133 \\
\hline 0.371 & 26.7644 & 0.514 & 26.8987 & 0.593 & 27.6948 & 0.125 & 27.8034 & 0.775 & 27.9156 \\
\hline 0.344 & 26.7667 & 0.559 & 26.9009 & 0.633 & 27.6971 & 0.123 & 27.8060 & 0.773 & 27.9179 \\
\hline 0.327 & 26.7691 & 0.574 & 26.9046 & 0.687 & 27.6995 & 0.110 & 27.8083 & 0.746 & 27.9204 \\
\hline 0.293 & 26.7729 & 0.654 & 26.9069 & 0.699 & 27.7018 & 0.115 & 27.8106 & 0.727 & 27.9227 \\
\hline 0.280 & 26.7752 & 0.736 & 26.9116 & 0.755 & 27.7041 & 0.120 & 27.8133 & 0.698 & 27.9250 \\
\hline 0.269 & 26.7775 & 0.732 & 26.9139 & 0.778 & 27.7064 & 0.122 & 27.8156 & 0.642 & 27.9278 \\
\hline 0.259 & 26.7807 & 0.788 & 26.9194 & 0.783 & 27.7087 & 0.113 & 27.8179 & 0.636 & 27.9301 \\
\hline 0.231 & 26.7830 & 0.781 & 26.9265 & 0.781 & 27.7135 & 0.134 & 27.8202 & 0.589 & 27.9324 \\
\hline 0.233 & 26.7853 & 0.712 & 26.9349 & 0.774 & 27.7158 & 0.133 & 27.8225 & 0.578 & 27.9352 \\
\hline 0.223 & 26.7889 & 0.694 & 26.9372 & 0.763 & 27.7181 & 0.132 & 27.8248 & & \\
\hline
\end{tabular}

$\mathrm{II}=2455326.92427 \pm 0.00068,2455327.91256 \pm 0.00060$. We also obtained the following timings of minimum light from parabola fits to the data of Blattler, 2006: HJD I = 2453382.6915, 2453445.4980, 2453502.3800, 2453515.4154, 2453517.3907; HJD II = 2453463.4719, 2453515.607. From these and Bob Nelson's timings, an improved ephemeris below was calculated from all the available eclipse timings:

J.D. Hel Min I = 2455326.9244 \pm 0.0005

$$
+0.39500870 \pm 0.00000016 d * E .
$$

The $\mathrm{O}-\mathrm{C}$ residuals are shown, graphically, in Figure 2 and tabled residualsare tabulated in Table 2 .

Observations taken over some 5000 orbits (5.3 years) seem to show a constant period. A quadratic ephemeris was calculated, but the negative quadratic term was not significant. Further observations are needed to determine any trends that indicate changes in period.

\section{Standard Magnitudes}

Observations of over a dozen Landolt standard stars along with the variable, comparison, and check stars throughout 
TABle 2: Times of minimum light, TYC 3034-299-1.

\begin{tabular}{|c|c|c|c|c|c|}
\hline NO. & JD Hel. EPOCHS & CYCLES & WEIGHT & $\mathrm{O}-\mathrm{C}$ & REF \\
\hline 1 & 53382.6915 & -4922.0 & 1.0 & -0.0001 & {$[2]$} \\
\hline 2 & 53445.4980 & -4763.0 & 1.0 & 0.0000 & {$[2]$} \\
\hline 3 & 53463.4719 & -4717.5 & 1.0 & 0.0010 & {$[2]$} \\
\hline 4 & 53502.3800 & -4619.0 & 1.0 & 0.0007 & {$[2]$} \\
\hline 5 & 53515.4154 & -4586.0 & 1.0 & 0.0008 & {$[2]$} \\
\hline 6 & 53515.6070 & -4585.5 & 1.0 & -0.0051 & {$[2]$} \\
\hline 7 & 53517.3907 & -4581.0 & 1.0 & 0.0011 & {$[2]$} \\
\hline 8 & 53936.4936 & -3520.0 & 0.5 & -0.0002 & {$[3]$} \\
\hline 9 & 54174.4898 & -2917.5 & 1.0 & 0.0032 & {$[3]$} \\
\hline 10 & 54547.7692 & -1972.5 & 1.0 & -0.0006 & {$[5]$} \\
\hline 11 & 54879.9715 & -1131.5 & 1.0 & -0.0006 & {$[5]$} \\
\hline 12 & 55326.7275 & -0.5 & 1.0 & 0.0006 & $\mathrm{PO}$ \\
\hline 13 & 55326.9242 & 0.0 & 1.0 & -0.0002 & $\mathrm{PO}$ \\
\hline 14 & 55327.7133 & 2.0 & 1.0 & -0.0012 & $\mathrm{PO}$ \\
\hline 15 & 55327.9125 & 2.5 & 1.0 & 0.0005 & $\mathrm{PO}$ \\
\hline
\end{tabular}

TABLE 3: Standard magnitudes of the variable, comparison and check stars.

\begin{tabular}{|c|c|c|c|c|c|c|}
\hline & $\mathrm{V}$ & $\mathrm{B}-\mathrm{V}$ & $\mathrm{V}-\mathrm{R}$ & R-I & V-I & mean \\
\hline VAR (phase 0.00) & $11.54(20)$ & $0.35(2)$ & $0.31(0)$ & $0.28(4)$ & $0.47(5)$ & \\
\hline Sp type & & $\mathrm{F} 2$ & F9 & F8 & $\mathrm{F} 4$ & F6 (3) \\
\hline VAR (phase 0.25) & $10.83(2)$ & $0.28(1)$ & $0.11(1)$ & $0.30(1)$ & $0.44(1)$ & \\
\hline Sp type & & F0 & A7 & G0 & F3 & $\mathrm{F} 2.5(5.5)$ \\
\hline VAR (phase 0.50) & $11.51(5)$ & $0.34(5)$ & $0.16(4)$ & $0.30(8)$ & $0.44(10)$ & \\
\hline Sp type & & $\mathrm{F} 2$ & F0 & F9 & F3 & F3.5 (4.0) \\
\hline VAR (phase 0.75) & $10.71(2)$ & $0.32(1)$ & $0.12(1)$ & $0.31(0)$ & $0.45(1)$ & \\
\hline Sp type & & $\mathrm{F} 1$ & A7 & G1 & F3 & F3 (6) \\
\hline COMP & $10.66(6)$ & $0.26(3)$ & $0.08(2)$ & $0.28(3)$ & $0.37(5)$ & \\
\hline Sp type & & F0 & A5 & F8 & $\mathrm{F} 1$ & F1 (5) \\
\hline $\mathrm{CHK}$ & $10.58(4)$ & $0.31(2)$ & $0.14(2)$ & $0.34(3)$ & $0.50(5)$ & \\
\hline Sp type & & $\mathrm{F} 1$ & F8.5 & G4 & F5 & F7.0 (5.5) \\
\hline
\end{tabular}

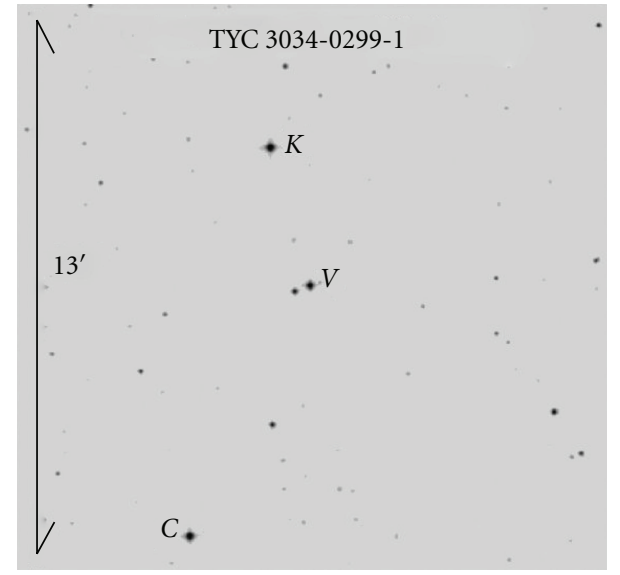

FIGURE 1: Finding chart, TYC 3034-299-1 variable $(V)$, comparison $(C)$ and check $(K)$. the evening of May 11 allowed us to calculate principal and transformation coefficients in BVR and I. Comparing these to Kron-Cousins calibrations, we found the results given in Table 3 [7] with the standard errors given in parentheses. From these calculations, we found a temperature of $6600 \mathrm{~K}$ (type F6V) for our primary star used for our synthetic light curve modeling.

\section{Light Curves}

The light curves were folded $\Delta \mathrm{U}, \Delta \mathrm{B}, \Delta \mathrm{V}, \Delta \mathrm{R}, \Delta \mathrm{I}, \Delta(\mathrm{U}$ B), $\Delta(\mathrm{B}-\mathrm{V})$, and $\Delta(\mathrm{R}-\mathrm{I})$ color curves using (2). They are given in Figures 3(a), 3(b), and 3(c). All bands give high precision, high amplitude $W$ UMa light curves with an obvious O'Connell effect due to spot activity. The curves reveal a time of constant light in the secondary minima 


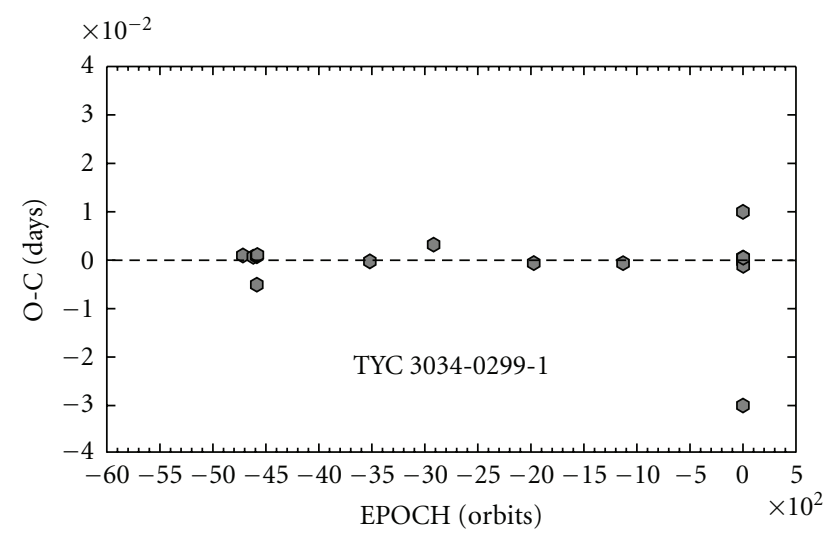

Figure 2: O-C residuals from (2).

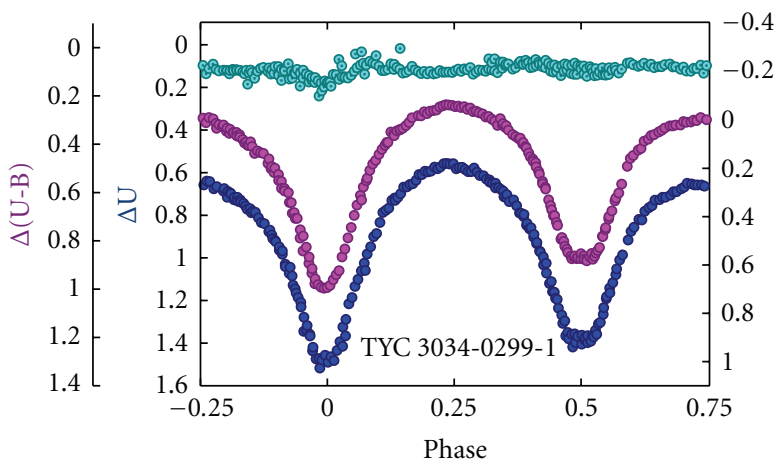

(a)

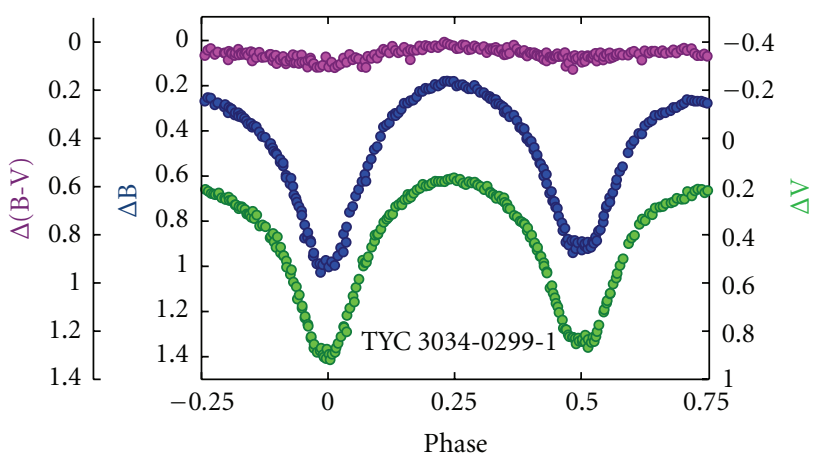

(b)

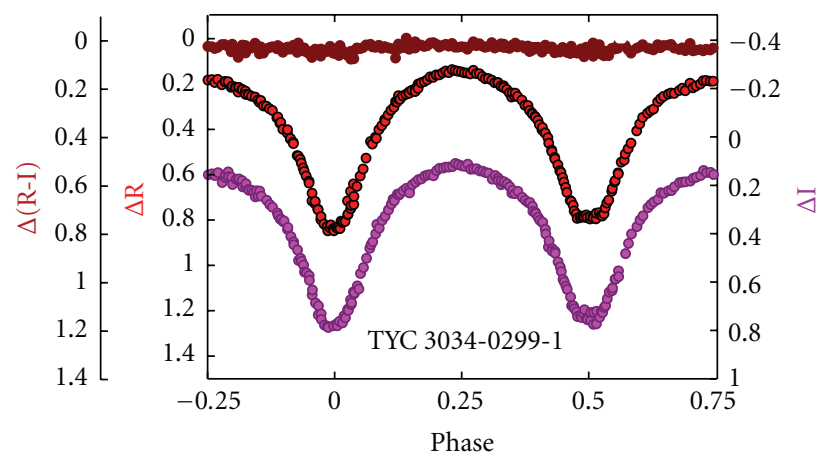

(c)

Figure 3: (a) U, B delta magnitude and color magnitudes versus phase plots in the sense of V-C. (b) B, V delta magnitude and color magnitudes versus phase plots in the sense of V-C. (c) R, I delta magnitude and color magnitudes versus phase plots in the sense of V-C.

indicating total eclipses. This usually indicates that the system is a $W$-type $W$ UMa binary system. The amplitudes are rather deep for a contact binary, ranging from $0.85 \mathrm{mag}$ in $\mathrm{U}$ to 0.66 in I. The O'Connell effect ranges from $67 \mathrm{mmag}$ to $36 \mathrm{mmag}$ from $\mathrm{U}$ to $\mathrm{I}$, respectively, revealing substantial magnetic activity.

\section{Synthetic Light Curve Solutions}

The U, B, V, R, and I curves were premodeled with Binary Maker 2.0 [8] fits in all filter bands. The parameters were then averaged and input into a 5-color simultaneous light curve calculation using the Wilson Code [9-12]. Adjusted parameters were those with parentheses in Table 4 .

The solution was computed in Mode 3, the contact mode. Convective parameters, $g=0.32, A=0.5$, were used. Our first solution, $q=0.52$, gave a sum of square residuals equal to 8.33 (goodness of fit parameter). This fit the out of eclipse shoulders well but did not match the eclipse at phase 0.50 . Due to the brevity of the total eclipse, the modeling procedure does not produce unambiguous results (many solutions may be possible). Consequently, we performed a 


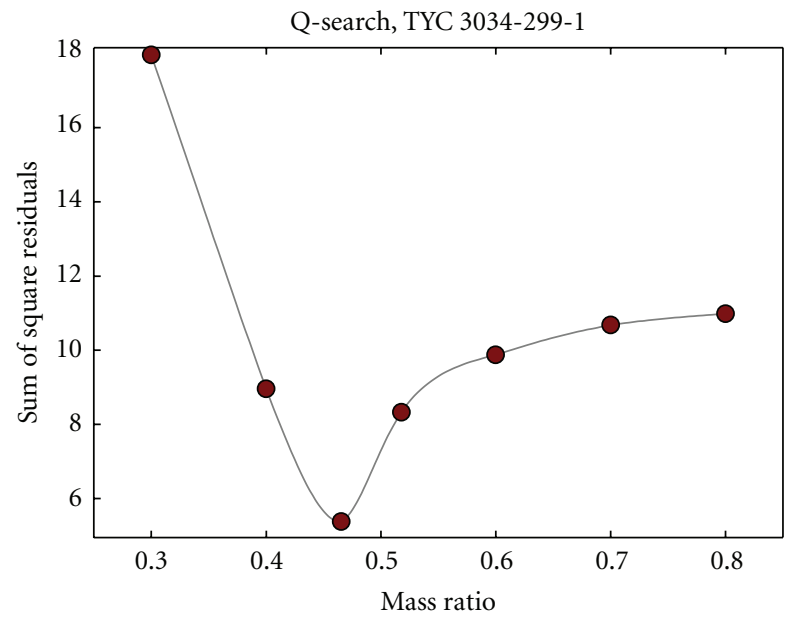

Figure 4: Determination of best fitting mass ratio.

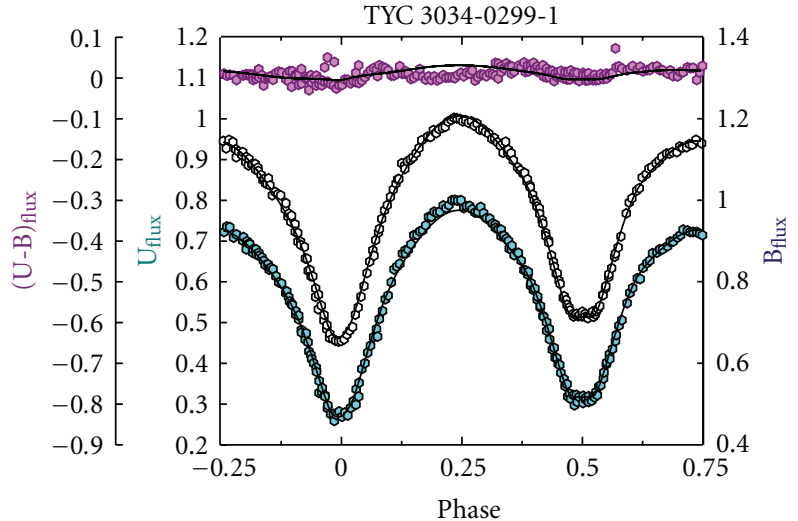

(a)

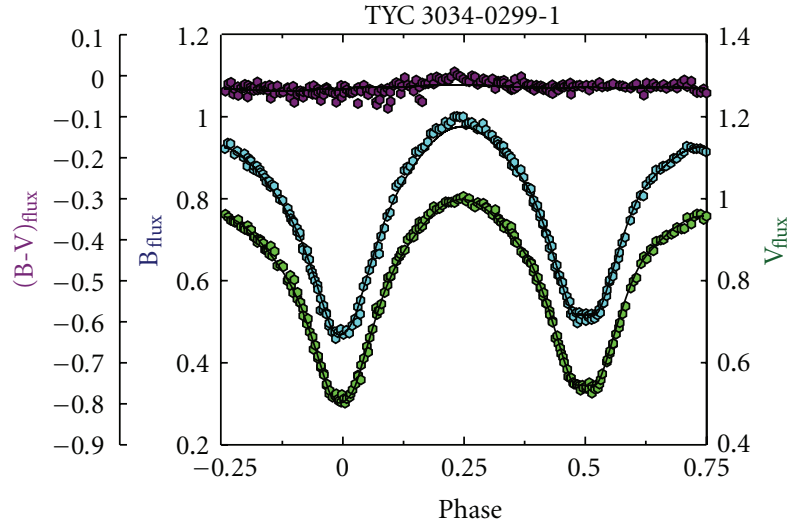

(b)

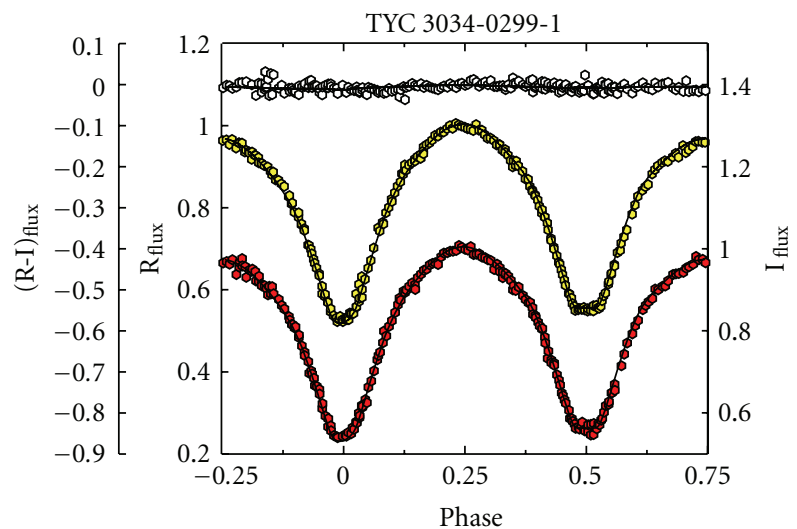

(c)

FIGURE 5: (a) U, B synthetic light curve solutions overlaying the normalized flux curves. (b) B, V synthetic light curve solutions overlaying the normalized flux curves. (c) R, I synthetic light curve solutions overlaying the normalized flux curves. 


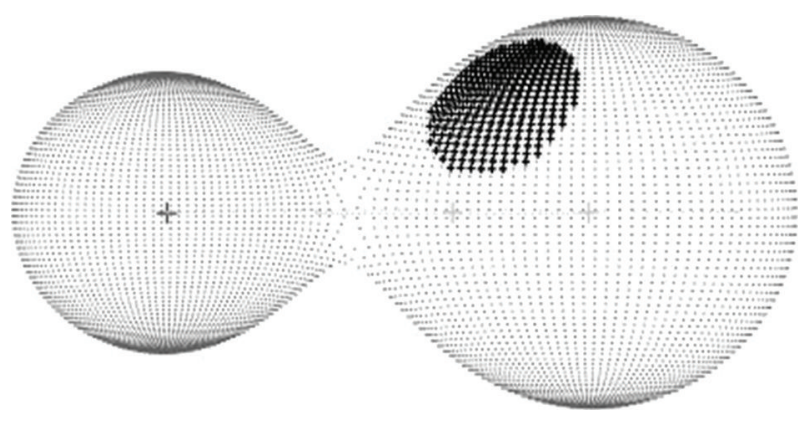

Phase 0.76 TYC 3034-299-1

(a)

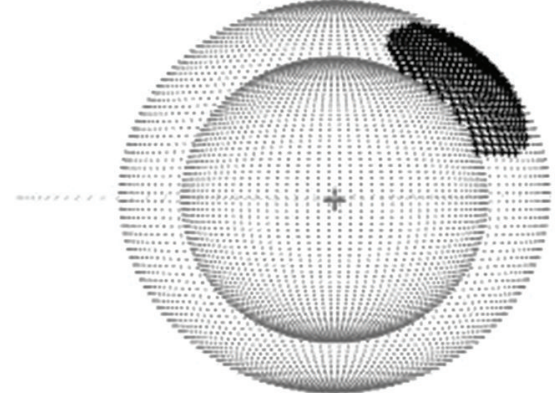

Phase 0 TYC 3034-299-1

(b)

FIgure 6: (a) Roche lobe surfaces from our UBVRI solution, phase 0.76. (b) Roche lobe surfaces from our UBVRI solution, phase 0.00.

TABLE 4: Synthetic curve parameters, TYC 3034-299-1.

\begin{tabular}{|c|c|}
\hline Parameters & Values \\
\hline$\lambda_{\mathrm{B}}, \lambda_{\mathrm{V}}, \lambda_{\mathrm{R}}, \lambda_{\mathrm{I}}(\mathrm{nm})$ & $360,440,550,640,790$ \\
\hline$x_{\text {boll }, 2}, y_{\text {boll }, 2}$ & $0.641,0.6410 .246,0.246$ \\
\hline$x_{1 \mathrm{I}, 2 \mathrm{I}}, y_{1 \mathrm{I}, 2 \mathrm{I}}$ & $0.541,0.5410 .276,0.276$ \\
\hline$x_{1 \mathrm{R}, 2 \mathrm{R}}, \quad y_{1 \mathrm{R}, 2 \mathrm{R}}$ & $0.625,0625,0.289,0.289$ \\
\hline$x_{1 \mathrm{~V}, 2 \mathrm{~V}}, y_{1 \mathrm{~V}, 2 \mathrm{~V}}$ & $0.794,0.7940 .263,0.263$ \\
\hline$x_{1 \mathrm{~B}, 2 \mathrm{~B}}, y_{1 \mathrm{~B}, 2 \mathrm{~B}}$ & $0.821,0.821,0.299,0.299$ \\
\hline$g_{1}, g_{2}$ & 0.32 \\
\hline$A_{1}, A_{2}$ & 0.5 \\
\hline Inclination $\left({ }^{\circ}\right)$ & $89.6 \pm 0.6$ \\
\hline$T_{1}, T_{2}(\mathrm{~K})$ & $6600 \pm 500,6695 \pm 3^{*}$ \\
\hline$\Omega_{1}=\Omega_{2}$ & $2.7645 \pm 0.0023$ \\
\hline$q\left(m_{2} / m_{1}\right)$ & $0.4777 \pm 0.0008$ \\
\hline Fill-outs: $F_{1}=F_{2}$ & $24 \%$ \\
\hline$L_{1} /\left(L_{1}+L_{2}\right)_{\mathrm{I}}$ & $0.6494 \pm 0.0005$ \\
\hline$L_{1} /\left(L_{1}+L_{2}\right)_{\mathrm{R}}$ & $0.6473 \pm 0.0009$ \\
\hline$L_{1} /\left(L_{1}+L_{2}\right)_{\mathrm{V}}$ & $0.6449 \pm 0.0009$ \\
\hline$L_{1} /\left(L_{1}+L_{2}\right)_{\mathrm{B}}$ & $0.6407 \pm 0.0007$ \\
\hline$L_{1} /\left(L_{1}+L_{2}\right)_{\mathrm{U}}$ & $0.6404 \pm 0.0007$ \\
\hline$J D_{o}$ (days) & $2455326.92478 \pm 0.00005$ \\
\hline Period (days) & $0.395014 \pm 0.000032$ \\
\hline$r_{1}, r_{2}($ pole $)$ & $0.4300 \pm 0.0012,0.3087 \pm 0.0017$ \\
\hline$r_{1}, r_{2}$ (side) & $0.4600 \pm 0.0016,0.3240 \pm 0.0022$ \\
\hline$r_{1}, r_{2}($ back $)$ & $0.4927 \pm 0.0023,0.3657 \pm 0.0041$ \\
\hline SPOT parameters & Dark Spot \\
\hline \multicolumn{2}{|l|}{ STAR 1} \\
\hline Latitude $\left({ }^{\circ}\right)$ & $55 \pm 1$ \\
\hline Longitude $\left({ }^{\circ}\right)$ & $51.3 \pm 0.8$ \\
\hline Spot radius $\left({ }^{\circ}\right)$ & $24.86 \pm 0.21$ \\
\hline Spot temperature & $5468 \pm 30$ \\
\hline
\end{tabular}

* All Errors are formal; here the error in $T_{2}$ is in relation to $T_{1}$. We expect errors to $T_{1}$ to be on the order of $\sim 500 \mathrm{~K}$ from the errors of our standard star determinations.

$q$-search over the interval from $q=0.3$ to 0.8 . The residuals minimized at $q \sim 0.45$ (see Figure 4). Additional iterations were run from the minimized $q$ with the mass ratio allowed to adjust to our final solution. Our final solution is a $W$-type
$W$ UMa binary, as noted from the light curve appearance. The solution is seen overlaying the normalized flux curves shown in Figures 5(a), 5(b), and 5(c). The complete solutions are given as Table 4 . Two phases of the Roche-lobe model of the binary for the dark spot solution are shown in Figures 6(a) and 6(b). Phase 0.0 shows the total eclipse.

\section{Discussion}

TYC 3034-299-1 is a mid- $F$ type magnetically active contact binary. The solution gives a eclipse duration of $\sim 7$ minutes. The firm $24 \%$ fillout and the nearly identical temperatures of the two stars show that the system has nearly reached thermal contact. After this point is reached, we would suspect that over long periods the mass ratio would become more extreme. This is caused by torques provided by stellar winds leaving the star along stiff magnetic field lines [13] rotating synchronously with the gravitationally coupled binary.

\section{Acknowledgments}

The authors wish to thank Lowell Observatory for their allocation of observing time, and the American Astronomical Society and the Arizona Space Grant for travel support for this observing run.

\section{References}

[1] C. Akerlof, S. Amrose, R. Balsano et al., "Rotse all-sky surveys for variable stars. I. Test fields," Astronomical Journal, vol. 119, no. 4, pp. 1901-1913, 2000.

[2] E. Blattler, "Observation of variables," Information Bulletin On Variable Stars, no. 5699, 2006.

[3] R. Diethelm, "List of timings of minima eclipsing binaries by BBSAG observers," Information Bulletin on Variable Stars, no. 5781, 2007.

[4] M. T. Geske, S. J. Gettel, and T. A. Mckay, "A rosat survey of contact binary stars," Astronomical Journal, vol. 131, no. 1, pp. 633-637, 2006.

[5] R. H. Nelson, "CCD minima for selected eclipsing binaries in 2008," Information Bulletin on Variable Stars, no. 5875, 2009.

[6] R. H. Nelson, "CCD minima for selected eclipsing binaries in 2009," Information Bulletin on Variable Stars, no. 5929, 2009. 
[7] A. N. Cox, Ed., Allen's Astrophysical Quantities, Springer, New York, NY, USA, 4th edition, 2000.

[8] D. H. Bradstreet and D. P. Steelman, "Binary maker 3.0an interactive graphics-based light curve synthesis program written in Java," Bulletin of the American Astronomical Society, vol. 34, p. 1224, 2002.

[9] R. E. Wilson and E. J. Devinney, "Realization of accurate closebinary light curves: application to MR cygni," Astrophysical Journal, vol. 166, pp. 605-619, 1971.

[10] R. E. Wilson, "Accuracy and efficiency in the binary star reflection effect," Astrophysical Journal, vol. 356, no. 2, pp. 613-622, 1990.

[11] R. E. Wilson, "Binary-star light curve models," Publications of the Astronomical Society of the Pacific, vol. 106, pp. 921-941, 1994.

[12] W. V. Van Hamme and R. E. Wilson, "Generalized solution for binary star ephemerides and apsidal motion," Bulletin of the American Astronomical Society, vol. 30, p. 1402, 1998.

[13] E. F. Guinan and D. H. Bradstreet, "Kinematic clues to the origin and evolution of low mass contact binaries, formation and evolution of low mass stars," in Proceedings of the NATO Advanced Study Institute, A. K. Dupree and M. T. V. T. Lago, Eds., vol. 241, pp. 345-375, NATO Advanced Science Institutes (ASI) Series C, Viana do Castelo, Portugal, September 1987. 

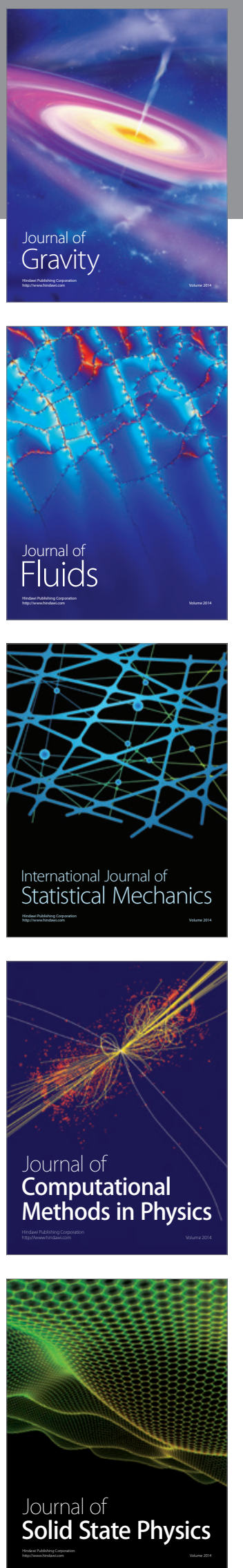

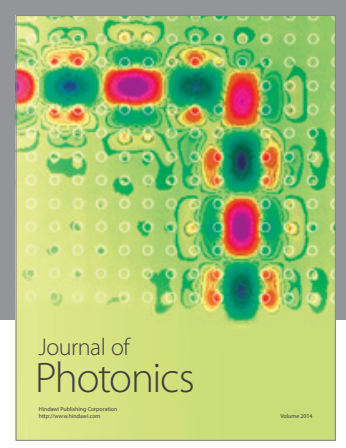

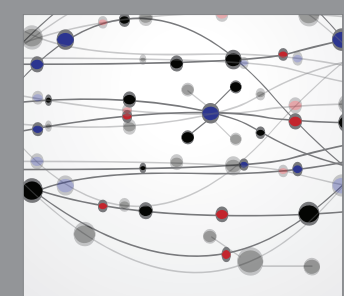

The Scientific World Journal
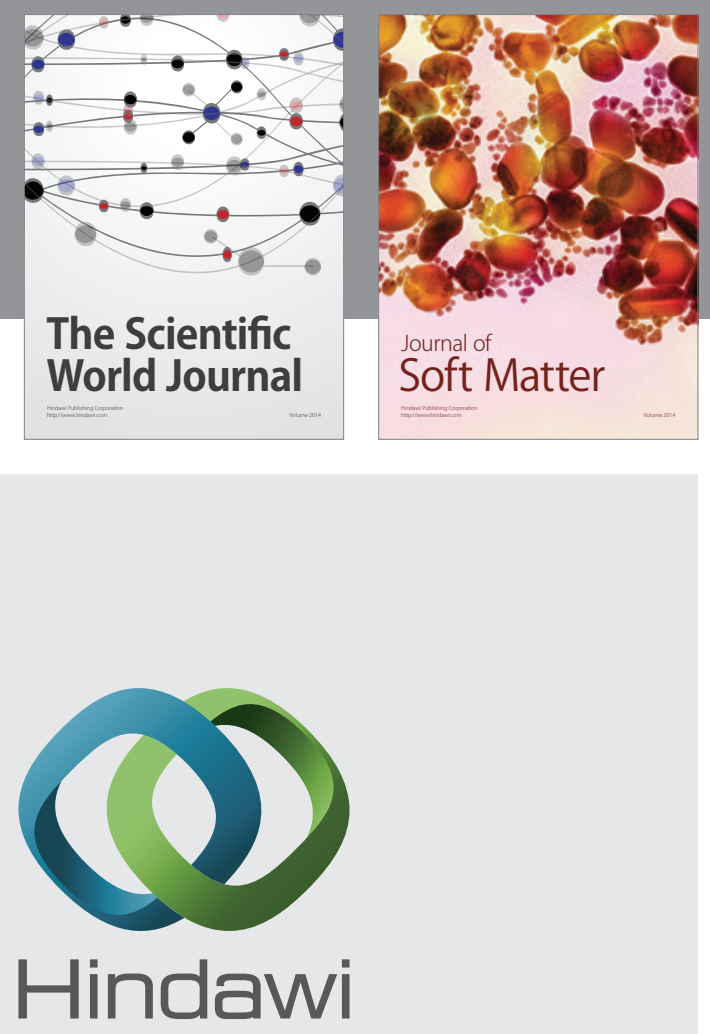

Submit your manuscripts at

http://www.hindawi.com
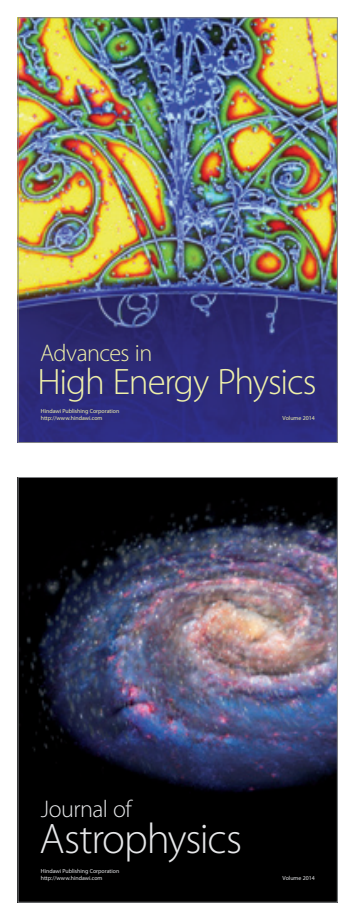
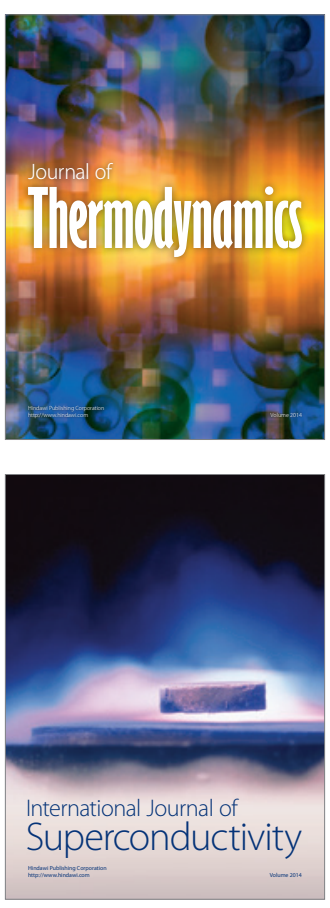
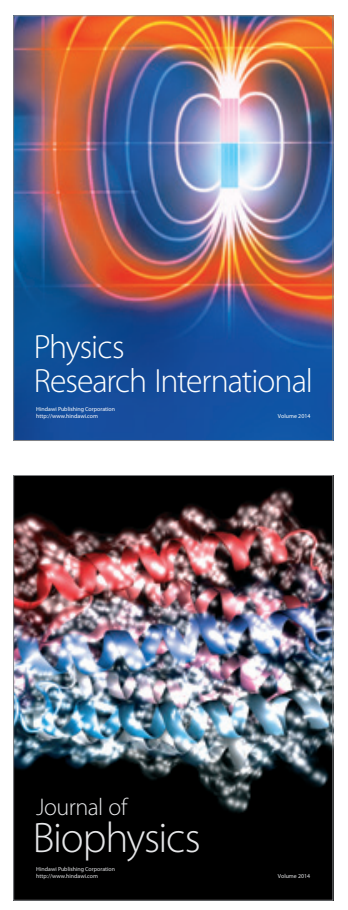
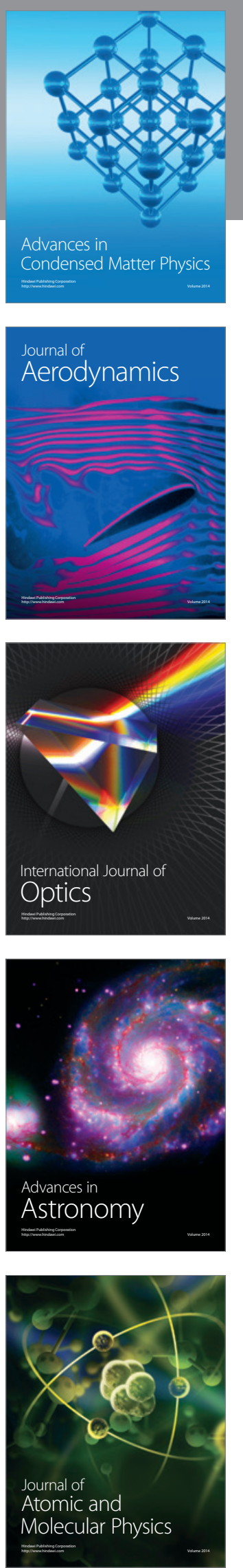